\title{
Wittgenstein on Colors and Internal Relations, 1930-1932
}

\author{
ANDREAS BLANK, TEL AVIV
}

\section{Introduction}

Colors play a major role in Wittgenstein's transitional period, roughly from 1930 to 1932. A considerable number of notebook and typescript entries from this period suggest the importance of colors, as well as some longer remarks in his conversations with the Vienna Circle. Many of these entries and remarks belong to the most opaque material from what is perhaps the least accessible period of Wittgenstein's philosophical development. After assembling the Big Typescript (1932/33), Wittgenstein put the issues connected with color aside, only to take them up again almost twenty years later, in the Remarks on Color (1950). While his views on color in the last years of his life have found widespread attention among his commentators, little work has been done on his views on color in the early 30s. The aim of the present paper is to shed some light on both the details and the overall strategy of what Wittgenstein, in the early 30s, has to say about color. In particular, it emphasizes the role of the concept of logical multiplicity (logische Mannigfaltigkeit) in Wittgenstein's approach to color and color representation in the period of 1930-32.

At various places in his notebooks of this period, Wittgenstein ascribes logical multiplicity to colors and color predicates. Obviously, the concept of a logical multiplicity is borrowed, more proximately, from his early philosophy and, more distantly, from the work of Helmholtz and Hertz (who derived it from Riemann). Helmholtz characterizes the concept as follows:

Riemann calls a system of differences, in which each particular can be specified by means of $n$ measures a n-fold extended multiplicity [n-fach ausgedehnte Mannigfaltigkeit] or a multiplicity of $\mathrm{n}$ dimensions. Thus the space known to us, in which we live, is a threefold extended multiplicity of points, a surface a twofold one, a line a onefold one, and time equally a onefold one. (Helmholtz 1977, 12)

The early Wittgenstein uses the concept of logical multiplicity to explicate the concept of internal relation. According to the Tractatus, internal relations hold between two states of affairs of the same logical multiplicity, 
e.g., between a gramophone record, the musical idea, the written notes, and the sound-waves. Relations of the same kind also hold between elementary propositions and states of affairs (see TLP 4.014). For the early Wittgenstein, having the same logical multiplicity involves two features: (1) the same number of simple, unanalyzable components (names, in the case of propositions, and simple objects, in the case of states of affairs) forming a configuration of the same structure, and (2) a logical space of possibilities of the same structure determined by the modal properties of the simple components-properties determining their possible configurations.

In the period of 1930-32, Wittgenstein modifies the Tractatus view of internal relations in a significant way. One of the decisive moments in his years of transition was that — on January 1, 1930-Wittgenstein came to the view that "the concept of elementary proposition now loses all its significance" (WA 2.158.4). Giving up the apparatus of elementary propositions and (elementary) state of affairs brings with it that he is no longer able to analyze logical multiplicities in terms of the number and configuration of simple constituents of propositions and facts. Moreover it brings with it that he no longer can analyze possibilities in terms of possible combinations of simple objects. On first glance, it might seem as if giving up the apparatus of elementary propositions and simple objects brings an end to the concept of logical multiplicity and, with it, to the concept of internal relations.

Nevertheless, in the period of 1930-32, Wittgenstein keeps on connecting the concept of a logical multiplicity to the concept of a space of possibilities. For example, red is embedded in a space to which, among others, yellow, orange, dark red, blue-red, and blue belong, but not red-green or blue-yellow. Likewise, a color predicate is embedded in a space of possible color predicates. According to Wittgenstein's view in the early 30s, a proposition describing a color is internally related to the described color if the logical multiplicity of the color predicate matches the logical multiplicity of the color. Intuitively, the idea seems to be that the logical multiplicity of a color predicate matches the logical multiplicity of a color if the color predicate is embedded in a space of possible color predicates in the same way as the color is embedded in a space of possible colors.

In which way did Wittgenstein think that different systems of color representation can have different logical multiplicities? To begin with, different systems of color representation can have different multiplicities in the sense that they involve more or less fine-grained distinctions between color predicates. A more technical question, to which Wittgenstein returns re- 
peatedly, is whether orange lies in the same sense between red and yellow as red lies between blue-red and orange (WA 2.213.6). He holds that if "lying in between" in the first case means "being a mixture of two pure colors" and in the second case "being a common component of two mixed colors", the multiplicity of the application of this expression would be different-a difference not of degree but of category (WA 2.219.2). Different systems of color representation give different answers to this question: using a double cone with a color circle in the middle and white and black at the tops does not differentiate between the two cases. Using such a system of color representation implies a conception of differences of color as gradual differences without marking any shade of color as "pure". Things are different with a system of color representation using a double 8-sided pyramid, since in this case the "pure" colors red, blue, green, and yellow are marked as points. Representing color by means of a color octahedron adds the relation of "lying in the middle between" two pure colors, since there also red-blue, blue-green, green-yellow, and orange are marked as points (see WA 2.213.6).

In the early 30s, Wittgenstein does not regard the multiplicity of a space of possibilities as something given independently of systems of representation. In particular, in the case of color he holds that there are no languageindependent formal properties of colors that necessitate a particular system of color representation (see PR 4). Rather, he maintains that the multiplicity of a given color depends on the multiplicity of the chosen system of representation. The aim of much of what follows in this paper is to explicate Wittgenstein's views on the role of conceptions in representing color and to make clear the motivations that stand behind his view that the multiplicity of systems of color representation is constitutive of the multiplicity in which we conceive colors.

\section{Internal Relations and Color Representation}

In his conversations with the Vienna Circle in 1930, Wittgenstein addresses the concept of internal relation and its application to the case of color in some detail. As he points out, "[t]he whole question of external and internal is tremendously confused." Nevertheless, he goes on to clarify some points about internal and external relations. He draws the following distinction: "A relation that says 'how?' is external. It is expressed by a proposition. 'Internal' - we have two propositions between which a formal relation holds" (WVC 54-55). This way of distinguishing between external 
and internal relations suggests that propositions can describe external relations, while relations between propositions exemplify internal relations. Yet there also are other examples of internal relations. Wittgenstein points out: "What I cannot say is that 2 is greater than 1.5. That is internal." By contrast, that one line is $0.5 \mathrm{~m}$ longer than another line is an external relation. What is external about this relation is explained as follows: "There I obviously have an external relation; for one can just as well imagine that line $a$ was shorter than $b$..." (ibid.). This way of arguing for the external character of the relation between two lines suggests that what makes a relation an external one is the possibility of imagining that the relata stand in a different relation to each other. While this is the case with two given lines, it is not the case with two given numbers: if the numbers 2 and 1.5 is given, it is unthinkable that the one is not greater than the other. That this is Wittgenstein's view of what makes a relation an internal one is confirmed by what he says about relations between different colors: "I cannot say that one color is darker than the other one. For this is of the essence of a color; without it, after all, a color cannot be thought" (WVC 55). Of course, one can imagine that two suits or two points in space have other colors than they actually have. That one suit is darker than another suit and that one point of space is darker than another are external relations between suits or points in space. As Wittgenstein puts it: "I have an external relation as soon as I bring in space; but between pure qualities of color only internal relations can obtain. After all, I have no other means of characterizing colors than by means of their quality" (ibid.). The last sentence in this quotation suggests that, since we have no other means of characterizing colors than by means of their quality, and since relations of being lighter or darker belong to the relations of these qualities, it is unthinkable that colors do not stand in these relations to each other.

Are internal relations between colors, according to the Wittgenstein of the early 30s, independent of the way we represent colors? Interestingly, the notion of color representation occurs in Wittgenstein's discussion of how colors form a system. Schlick asks Wittgenstein to consider the following case:

How would it be, for example, if a person was locked in a red room for his whole life and could not see any color but red? Or if a person's entire visual field contained only a uniform red? Could he then say to himself, 'I see only red; but there must also be other colors'? (WVC 65) 
Schlick's concern is whether the claim that colors form a system is an empirical or an a priori claim, i.e., about whether the system of colors presupposes a system of color experiences. Wittgenstein responds that, in order to know the system of colors, it is unnecessary to ask how many colors a person must have experienced. He compares the case of colors to the case of space, where even a person locked in a room knows that space extends beyond this room, even if this is not a matter of experience. According to Wittgenstein, the possibility of being outside the room is an a priori part of what he calls the "syntax of space" (WVC 65-66). Making the possibilities of being located in space dependent on the syntax of propositions about spatial locations suggests that, in Wittgenstein's view, the structure of space is not independent of the system of spatial representation. Rather, the possibility of being outside the room is presupposed by the possibilities of specifying spatial locations inherent in the system of spatial representation. In this sense, the logical space of possible spatial locations is not independent of the logical space of possible specifications of spatial locations.

Something analogous holds for the way colors form a system. In the case of the person in the red room, Wittgenstein distinguishes between two cases: The first case is that the syntax of the person in the red room is the same as ours, involving, e.g., distinctions expressed by means of predicates such as "red", "redder", "bright red", "yellowish red", etc (and, presumably, the exclusion of predicates such as "red-green"). In this case, Wittgenstein holds that this person has our complete system of colors. The second case is that her syntax is not the same. In that case, Wittgenstein holds that she does not know even a single color in our sense at all. As he summarizes his view: "The crucial point is not how many colors one has seen, but the syntax" (WVC 66-67). Tying the system of colors in this way to the syntax of color indicates that Wittgenstein regards the system of colors as involving a system of color representation.

The analogy between the syntax of spatial representation and the syntax of color representation indicates that a space of possibilities plays a similar role in both cases. Indeed, Wittgenstein uses the notion of logical multiplicity to characterize how colored patches on a surface are represented:

[T] he description will contain equations for lines and indices for colors, these elements of description are necessary, i.e. every possible description must have this multiplicity. The description may also be incomplete. I say for example, 'The inside of a certain patch is blue, outside it the paper is partly white, partly black' (WVC 75). 
These remarks indicate that propositions about colors possess a logical multiplicity. The relevant multiplicities of sentences describing colors or lengths are in the dimension of the space of possible specifications of color. Moreover, the logical space of color specifications presupposes the existence of a logical space of colors. Consider the following passage:

[I]f everything I saw were red and I could describe it, then I should also have to be able to construct the proposition that it was not red. This presupposes the possibility of other colors. Or else red is something that I cannot describe- then I have no proposition either and there is nothing I can deny ... (WVC 88-89)

Describing a color presupposes the possibility of other, incompatible descriptions of color, and the possibility of other descriptions presupposes the possibility of other colors. In this sense, both propositions about colors and colors are in a space of possibilities. Moreover, the concept of a space of possibilities clarifies the concept of a system of colors: Colors form a system since each color is embedded in a space of other possible colors. This is why Wittgenstein holds that seeing any color presupposes the possibility of red (WA 2.92.1).

\section{Internal Relations and Intermediary Colors}

Introducing the concept of a system of colors as what is presupposed by the syntax of color representation provides Wittgenstein with a characterization of the internal relations between a proposition describing a color and the color: the proposition and the color are internally related in case the proposition and the color are part of systems with the same logical multiplicity. However, if the multiplicity of a given system of colors is thought of only in terms of the number of possible colors, any color within the system has the same multiplicity as any other color in the same system. Likewise, if the multiplicity of a given system of color representation is thought of only in terms of the number of possible color predicates, any predicate has the same multiplicity as any other predicate in the same system. But, even if the same number of possibilities is a necessary condition for color representation, it cannot be a sufficient condition, since in this case any predicate in a given system of color representation could represent any color in a color system of the same multiplicity. Indeed, Wittgenstein has a richer notion of the logical multiplicity of color and color predicates. In particular, the possible relations between color predicates are a defining characteristic of the multiplicity of a system of color representation. Like- 
wise, the possible relations between colors are a defining characteristic of the multiplicity of a color system. This becomes clear in Wittgenstein's discussion of the relation of "lying in between" two colors.

In his notebooks of 1930-31, Wittgenstein repeatedly discusses the question of whether orange lies in a different sense in between red and yellow than red lies in between orange and red-blue. In notebook entries of 1930, he considers two plausible ways of thinking about the relations of "lying in between". One of them is to think of the mixture of two colors in a way such that the resulting intermediate color contains the two colors as components (Bestandteile) (see WA 2.214.2). In fact, thinking about internal relations between colors in this way is closely similar to Helmholtz's conception of the logical multiplicity of color, and although Wittgenstein does not mention this conception explicitly, the physiological tradition inspired by Helmholtz might well be the background against which Wittgenstein develops his own, diverging, view. Helmholtz holds that the colour system consists of a three-fold multiplicity, insofar as every colour can be represented as a mixture of a certain quantity of the three basic colors. Moreover, he maintains that by using a colored spinning top, one can actually carry out such measurements (Helmholtz 1977, 12). Indeed, Wittgenstein embraces such a view of the mixture of colors at least once, at an early stage of his conversations with the Vienna Circle: "Whatever color I see, I can represent each of them by mentioning the four elementary colors red, yellow, blue, green and adding how this particular color is to be generated from the elementary colors" (WVC 42). However, in his notebooks, he soon becomes skeptical about a conception of mixture in terms of common components. In particular, he realizes that the mixture of pigments is irrelevant for his investigation because, as he points out, this way of thinking about intermediary colors only indicates the way in which the pureness of a color could be defined as an external property (see WA 2.215.1). By contrast, Wittgenstein points out: "To see that pure color is not a property-external property-of a color means to see that I couldn't think that-e.g.- - purple has this color or that pure blue doesn't have it" (WA 2.220.4). But if mixture is not understood in terms of a mixture of pigments, it becomes questionable whether a conception of mixture in term of common components of a color along the lines suggested by Helmholtz makes sense. Wittgenstein remarks: "Does gray have something of black in the same sense as black of gray?! Obviously not, for I can get from white through gray to black but not from gray through black to white" (WA 2.214.5). There are cases in which a conception of intermediate colors as 
having the same components as the colors they lie in between does not make sense. This leaves Wittgenstein with the question of whether there are cases in which it does make sense to say that a color has other colors as components: "The question is whether what is relevant for the internal relation between two colors are only the pathways by means of which they can be transformed into each other" (WA 2.214.10). He considers the following case:

In addition to the transition from color to color on the color circle, there appears to exist a certain different one, with which we are confronted when we see small patches of the one color mixed with small patches of the other color. Of course, I here mean a seen transition.

And this kind of transition gives to the word "mixture" a new meaning, which does not coincide with the relation 'between' on the color circle. (WA 2.215.2)

One case in which an analysis of an intermediate color in terms of a mixture of small patches of pure colors seems to make sense is the case of orange. However, Wittgenstein objects, "if I say, in the ordinary sense, that red and yellow yield orange, nothing is said about the quantity of components" (WA 2.217.3). He goes on to explain: "[I]f a certain orange is given, I can't say that more red would have made it a redder orange (I don't talk about pigments), although of course it makes sense to speak of a redder orange. But it does not make sense, e.g., to say that this orange and this purple contain the same amount of red. And how much red would red contain?" (WA 2.217.3). His objection seems to be that a quantitative and compositional analysis along the lines suggested by Helmholtz does not have anything to do with the semantics of ordinary propositions about color. And holding on to an analysis of color that does not have anything to do with our ordinary way of speaking is contrary to Wittgenstein's view that "analysis is analysis of something that we have; not of something that we do not have" (WA 2.158.3). But, then, what are we to make of such patently quantitative expressions such as "add more red"? Wittgenstein suggests the following solution:

The comparison that one is erroneously tempted to make is the one between the color series with a system of two weights on a scale such that by means of augmenting or shifting the weights I can move the point of gravity of the system ...

\footnotetext{
1 "the relation" inserted by Wittgenstein.
} 
[D]oes it mean anything to say, more of this red? ... It can only mean something if I understand with pure red a certain previously assumed number of units. But then, the full number of these units does not mean anything but that the cup stands on red. By means of ratio numbers, again only a place on the scale is specified but not a place and a weight. (WA 2.218.1)

Although this passage is far from being crystal clear, Wittgenstein's point seems to be that specifying the number of units of a particular color does not make sense, not does it specify the number of components of a given intermediate color. Rather, all that can be achieved by means of a quantitative specification such as "more of this red"-meaning, presumably, more of a particular shade of red-is to specify a place on the color scale. In this case, such quantitative specifications implicitly involve the reference to a particular system of color representation, such as the color scale. The internal relation holding between two colors and an intermediate color, then, is not a relation that involves common components but rather relative positions in a particular system of color representation. Thus, in the case of orange the only sense of "mixture" is the one that coincides with the relation in lying in between red and yellow on the color scale.

In a set of notebook entries of 1931, Wittgenstein comes back to internal relations between colors. He writes:

It must lie in the essence (in the grammar) ${ }^{2}$ of this red shade that a more or less of it is possible; a reddish blue can be more or less close to pure red and hence, in this sense, contain more or less red. The proposition that specifies that red, as an ingredient of a color, is present here would have to mention ${ }^{3}$ somehow a quantity of red; but in this case, the proposition would have to have sense even apart from the logical product, and it would have to make sense to say that this place is colored purely red and contains ${ }^{4}$ this and that quantity of red, and this does not make sense. (WA 4.230; BT 100)

Thus, quantitative statements about colors such as red are not about the quantity of components of, e.g., pure red. Rather, that a given color, such as reddish blue, can contain more or less red is a matter of the grammar of color statements. Again, this suggests that quantitative statements about colors specify a particular position in a system of color representation.

His rejection of a view of internal relations between colors in terms of common components makes clear in which sense Wittgenstein thinks

\footnotetext{
2 "(in the grammar)" inserted by Wittgenstein.

${ }^{3}$ First variant: "specify".

4 "contains" underlined by Wittgenstein with a wavy line.
} 
about relations between colors as structural relations. He writes: "A mixed color, or rather intermediate color, of blue and red is mixed by means of an internal relation to the structures of blue and red." The structures of blue and red, however, are not to be understood in terms of a quantitative specification of common components but rather in terms of the grammatical relations between color predicates: "Expressed more correctly: what we call an 'intermediate color of blue and red' (or 'blue-red'), is called so due to a kinship that shows itself in the grammatical determinations about the words 'blue', 'red' and 'blue-red'." He explains: "The proposition that talks about an internal relation of the structures already arises from an incorrect conception; from the one, which sees in the concepts red, blue, etc. complicated structures (buildings ${ }^{6}$ ); which we at first see from the outside and the inner construction of which analysis must show ${ }^{7}$." If intermediate colors are not analyzed in terms of common components, there is no sense in which colors can have a compositional structure. Hence, also internal relations between colors cannot be understood as relations between compositional structures. As an alternative to a compositional analysis of colors, Wittgenstein suggests the following:

[T]he kinship (Verwandtschaft) between the pure colors and their intermediate color is of an elementary kind; i.e., it does not consist in this that the proposition which ascribes to an object the color blue-red consists of the propositions which ascribe to it the colors red and blue. And, hence, also the kinship between different degrees of a reddish blue, e.g., is an elementary kinship. (WA 4.238.3)

Moreover, in the first two variants of this passage, Wittgenstein rejects the view that the proposition ascribing the intermediate color is "the (or a) logical product of the propositions" ascribing the pure colors. Because the proposition ascribing the intermediate color does not display the complexity of a logical product of propositions about pure colors, so the argument seems to go, the intermediate color, too, cannot display the complexity required in order to be analyzable in terms of similarities as to compositional structure. The relation of an intermediate color to the colors it lies in between is an elementary relation in the sense that it involves the relation of the predicate of the intermediate color to the color predicates it lies in between in a chosen system of color representation. This how, in the case of

\footnotetext{
${ }^{5}$ First variant: "in the grammar of the words".

${ }^{6}$ First variant: "mechanisms".

${ }^{7}$ First variant: "will reveal".

8 "also" inserted by Wittgenstein.
} 
intermediary colors, the multiplicity of the system of color representation is constitutive of the multiplicity of the system of colors.

\section{REFERENCES AND ABBREVIATIONS}

Helmholtz, H. v. 1977 "On the Origin and Significance of the Axioms of Geometry", in H. von Helmholtz, Epistemological Writings, Dordrecht, Boston: Reidel, 1-26.

Waismann, F. 1979 Wittgenstein and the Vienna Circle, ed. B. F. McGuinness, Oxford: Blackwell [=WVC].

Wittgenstein, L. 1974 Tractatus Logico-Philosophicus, tr. D. F. Pears and B. F. McGuinness, London, Henley: Routledge and Kegan Paul [=TLP].

Wittgenstein, L. 1975 Philosophical Remarks, tr. R. Hargreaves and R. White, Oxford: Blackwell, 1975 [=PR];

Wittgenstein, L. 1993 Wiener Ausgabe, ed. M. Nedo, Vienna, New York: Springer $[=\mathrm{WA}]$. 
